\title{
Ultrasound plays a key role in imaging and management of genital angiomyofibroblastoma: a case report
}

\author{
Benjamin Wolf ${ }^{*}$, Lars-Christian Horn ${ }^{2}$, Romy Handzel ${ }^{1}$ and Jens Einenkel ${ }^{1}$
}

\begin{abstract}
Introduction: Angiomyofibroblastoma is a benign, rare mesenchymal tumor arising from the genital tract of both men and women and was first described by Fletcher and colleagues in 1992. The tumor needs to be distinguished from other, similar lesions, such as deep and superficial aggressive angiomyxoma and cellular angiofibroma, because aggressive angiomyxoma demands much more extensive treatment. The vast majority of angiomyofibroblastomas arise from the vulva and appear as solid cystic masses on ultrasound images.

Case presentation: We report a case of a 35-year-old Caucasian woman with an angiomyofibroblastoma arising from the vagina. She presented with a painless mass of about $5 \mathrm{~cm}$ in diameter that had a rather homogeneous, hypoechoic appearance on ultrasound images. The patient underwent surgical resection of the mass, which was subsequently diagnosed as angiomyofibroblastoma. We present sonographic and magnetic resonance imaging findings, intraoperative and histologic images, and a thorough review of the literature.

Conclusions: In our opinion, ultrasonography is the most valuable tool to establish a preoperative diagnosis of this tumor entity, differentiate it from other lesions of the female genital tract, and plan surgery accordingly. Even though it is a rare tumor, gynecologists should be able to recognize it and to differentiate it from other tumor entities that demand more aggressive treatment. We describe a different sonographic appearance of this tumor than previously reported.
\end{abstract}

Keywords: Angiomyofibroblastoma, Transvaginal ultrasound, Mesenchymal tumors of the lower genital tract

\section{Introduction}

Angiomyofibroblastoma (AMFB) was first described by Fletcher and colleagues in 1992 as a distinct subgroup of mesenchymal tumors histologically similar to aggressive angiomyoma (AA) that usually arise from the genital tract in both men and women [1]. The vast majority of cases originate from the vulva (Fig. 4a). One case of AMFB in the nasal cavity has been described recently [2].

In contrast to AA, which is characterized by local destructive growth and recurrence after clear margin resection in up to $47 \%$ of cases [3-5], AMFB has an excellent prognosis. Clinically, the tumor has well-circumscribed

\footnotetext{
* Correspondence: benjamin.wolf@medizin.uni-leipzig.de

'Department of Obstetrics and Gynecology, Leipzig University, Liebigstrasse 20a, 04103 Leipzig, Germany

Full list of author information is available at the end of the article
}

margins, a size of usually about $5 \mathrm{~cm}$ at the time of diagnosis, and virtually no tendency for local recurrence after excision. Histologically, these tumors are characterized as being composed of plump, ovoid, or, less often, spindleshaped cells, with limited eosinophilic cytoplasm and ovoid nuclei $[1,6]$. Characteristically, the stroma contains many capillary-sized blood vessels, around which the characteristic cells aggregate [6].

Together with cellular angiofibroma $(\mathrm{CA})$ and myofibroblastoma (MFB), AMFB belongs to a group of benign stromal tumors of the lower female genital tract. These tumors, along with AA, have significant overlap in their morphological and immunohistochemical characteristics, which can pose serious diagnostic problems [7]. Recent research, however, has shown that a common cytogenetic aberration present in angiofibroma (AF) and MFB (loss of 
the 13q14 region) is not shared by $A M F B$, suggesting that AMFB is not genetically related to AF and MFB [7].

\section{Case presentation}

A 35-year-old Caucasian woman was referred to our outpatient clinic for evaluation of a neoplastic lesion located adjacent to the uterine cervix. She reported not having experienced any pain or discomfort with regard to the lesion and that she had not noticed any changes in bladder or bowel function. The lesion had come to her gynecologist's attention during a routine pelvic examination 1 week earlier. Her history was remarkable for the excision of a melanoma from her right inner thigh about 4 years earlier. Two inguinal lymph nodes that were excised for sentinel staging at the time were reportedly without metastasis.

In her gynecological examination at our clinic, no inguinal lymph node swelling was noted, and her vulva and proximal vagina appeared normal. The vaginal mucosa on the right side of the posterior fornix was bulging inward, displacing the uterine cervix laterally to the left. Underlying the mucosa was a palpable, semimobile mass of about $5 \mathrm{~cm}$ in diameter that was of plump, elastic consistency. The vaginal mucosa overlying the tumor
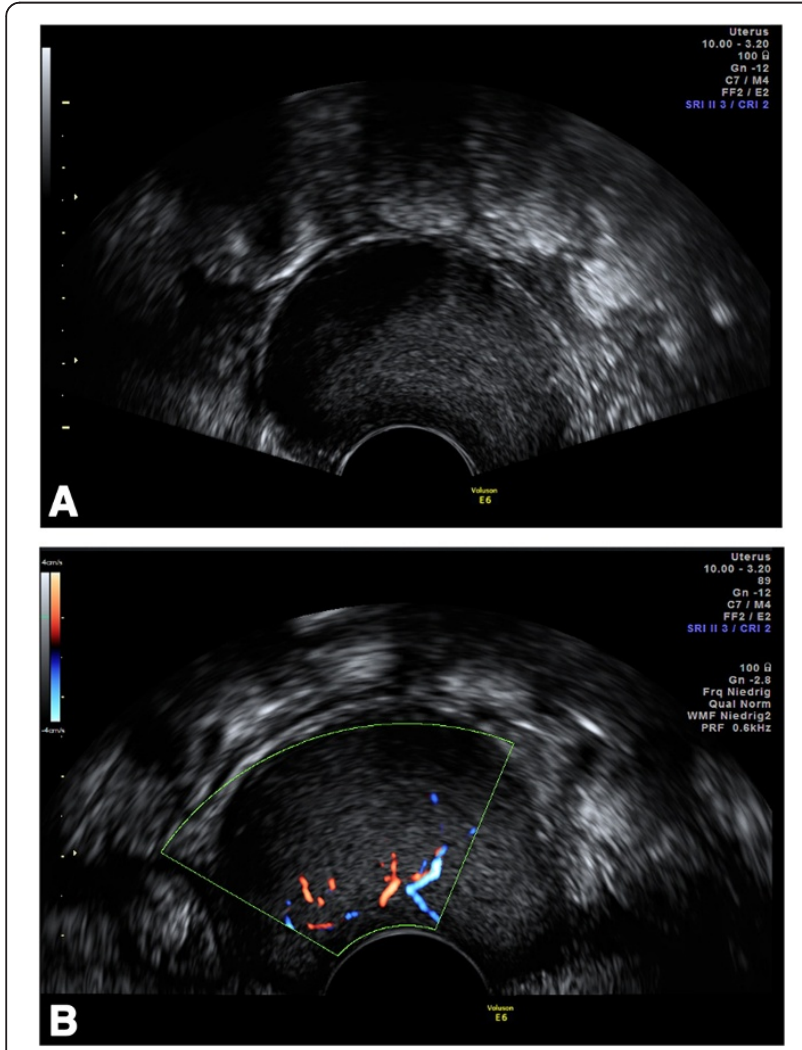

Fig. 1 a Transvaginal ultrasound image shows a well-demarcated, homogeneous mass of medium echogenicity (sagittal view). Intralesional septations can be seen only in Additional file 1. b Color Doppler imaging reveals intralesional vascularization was smooth but not mobile relative to the tumor. There were no signs of ulceration or retraction by the tumor.

Sonographically, the mass appeared homogeneous with medium echogenicity, few septations, and smooth edges (Fig. 1), and it had no papillary projections. Color Doppler imaging revealed several vessels visible within the structure. Subsequently, we took a core biopsy specimen of the tumor while the patient was under general anesthesia. A pathological examination revealed a mesenchymal tumor, most probably benign owing to the absence of any mitotic figures; however, it was not possible to classify the tumor any further. We discussed the findings with the patient and recommended surgical excision of the tumor. Owing to the unusual location of the tumor, we obtained additional magnetic resonance (MR) images of the pelvis (Fig. 2). T1- and T2-weighted images showed a homogeneously hypointense lesion that led to deviation of the cervix and the rectum. For excision, we performed a horizontal colpotomy about $1.5 \mathrm{~cm}$

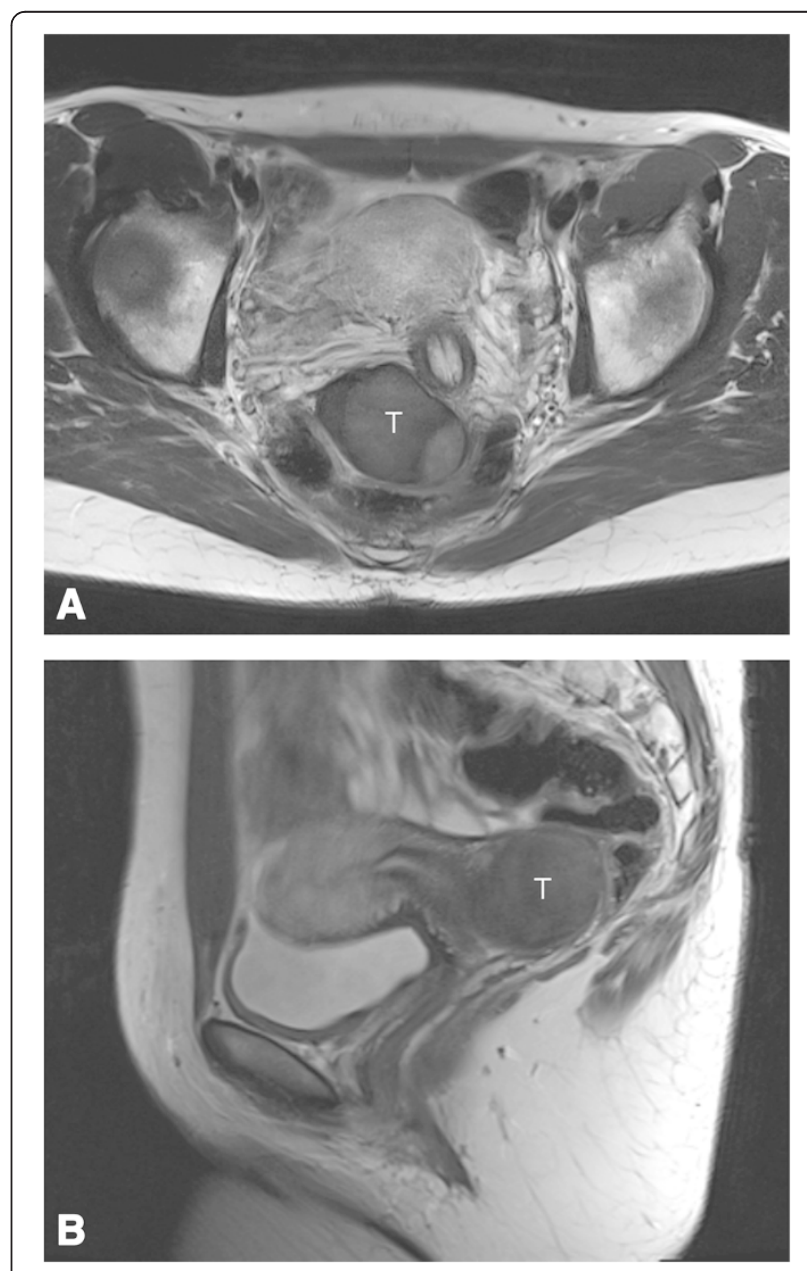

Fig. 2 Coronal (a) and sagittal (b) T2-weighted magnetic resonance images obtained preoperatively show a homogeneous solid mass ( $T$ ) displacing the cervix at left (seen best in coronal view) 
distant from the cervix, including the biopsy canal. The tumor appeared encapsulated and was dissected from the surrounding tissue. Gross examination of the tumor showed a $9 \times 6 \times 1.5-\mathrm{cm}$ pinkish gray mass weighing $65 \mathrm{~g}$. The tumor was covered entirely with a frail, wellvascularized membrane (Fig. 3a).

Histologically, the highly cellular lesion represented varying cellularity with vascularization by thin-walled blood vessels surrounded by spindle-shaped epithelioid cells with abundant eosinophilic cytoplasm and positive immunostaining for desmin and estrogen receptor (Fig. 3c, d).

The mesenchymal cells showed a diffusely positive reaction to $\mathrm{CD} 34$, and $70 \%$ stained positive for desmin.
There was no staining reaction to smooth muscle actin and S100. About $90 \%$ of the cells were estrogen receptorpositive. On the basis of these characteristics, a diagnosis of AMFB was made.

At her follow-up examination 17 months postoperatively, the patient was doing well and without any evidence of recurrent disease or sexual or urinary dysfunction.

\section{Discussion}

Including our patient, 137 cases of AMFB have been reported to date (for a detailed list, see Additional file 2). The median age of all patients at the time of presentation was 45 years (Fig. 4b), the majority of the patients
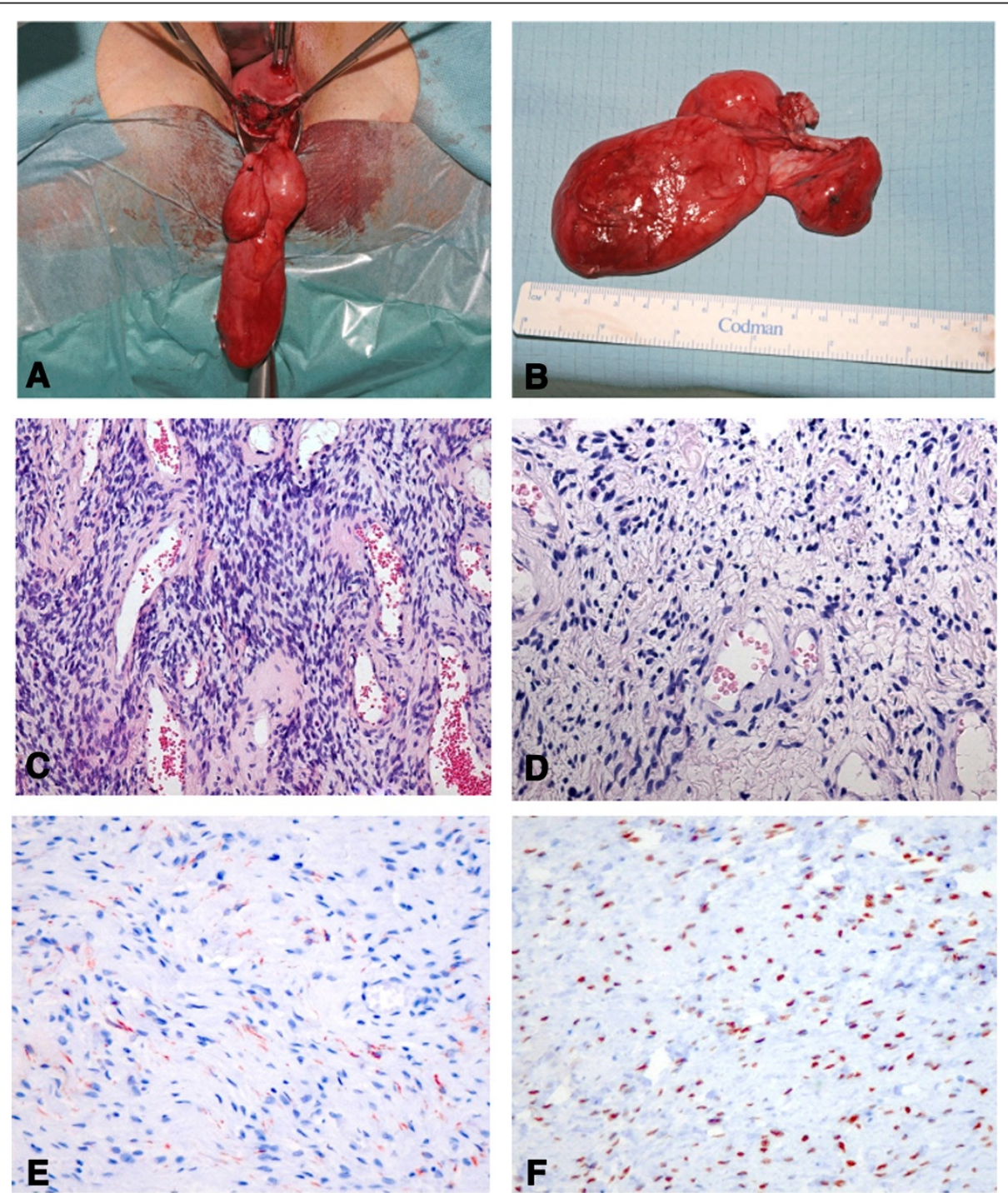

Fig. $\mathbf{3} \mathbf{a}$ and $\mathbf{b}$ Intraoperative appearance of the tumor. The tumor was covered entirely with a frail membrane of pinkish gray appearance. c Cellular mesenchymal lesion with alternating cellularity intermingled with small blood vessels [hematoxylin and eosin (H\&E) stain, original magnification $\times 109$ ]. $\mathbf{d}$ Higher-magnification image representing thin-walled blood vessels surrounded by ovoid to spindle-shaped cells with some epithelioid appearance and abundant eosinophilic cytoplasm (H\&E stain, original magnification $\times 241$ ). e Immunohistochemical staining for desmin with weak positivity. $\mathbf{f}$ Strong nuclear expression of estrogen receptor. 


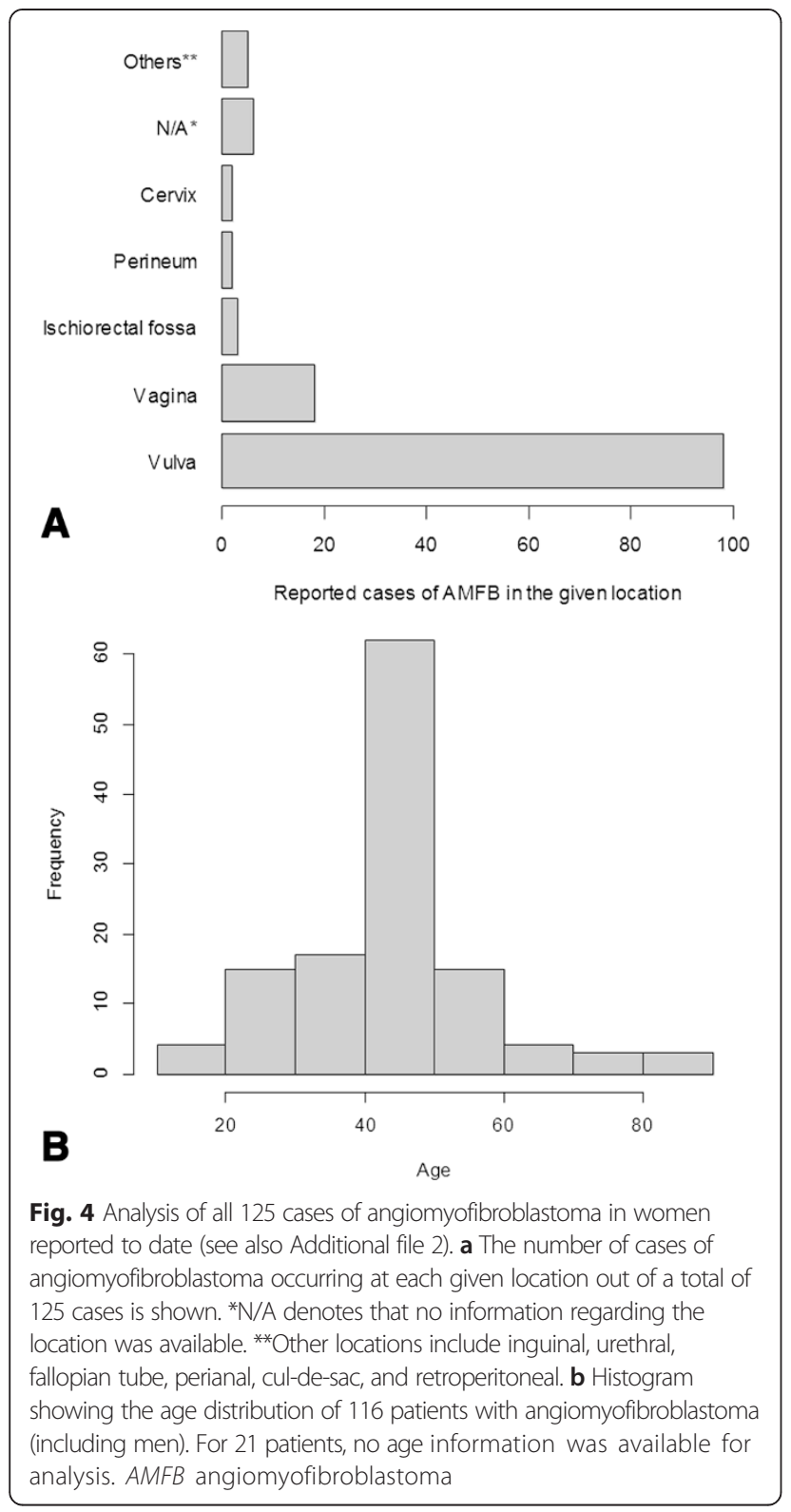

had been aware of their tumors for about 1 year. No recurrences have been reported after excision, although it has to be pointed out that the median follow-up of all cases published thus far is only 12 months. (Information about follow-up was available for only $64 \%$ of published case reports; see Additional file 2). Only one case of sarcomatous transformation has been reported; however, there was no recurrence after resection in that patient [8]. We found the female-to-male ratio to be 10:1. In women, the vast majority of tumors are located in the vulva (Fig. 4a). Owing to their low frequency, AMFBs and other vaginal soft tissue tumors can easily be confused clinically or sonographically with other, more common vaginal masses, such as Bartholin's cyst, rectocele, or urethral diverticulum [6,9-11]. However, soft tissue tumors can be differentiated from these masses by demonstrating intralesional vascularization using color Doppler imaging. Once this has been accomplished, echogenicity and demarcation of the tumor can help to further establish the diagnosis.

Sonographically, AMFB has been reported to be well demarcated with inhomogeneous echogenicity and multiple hypoechoic areas within an echogenic stroma [12]. Wang and coworkers assessed 72 perineal tumors, two among which represented AMFB and were characterized as solid cystic masses on the basis of ultrasonography [13]. In contrast to these descriptions, we found the tumor in our patient to be of homogeneous, medium echogenicity without solid cystic features (see Additional file 1). Retrospectively, the obtained MR images did not add much information to what was already known based on the ultrasound studies.

To date, the exact pathogenesis of AMFB is not clear. Because many tumors express estrogen and progesterone receptors, it is likely that these hormones play a crucial role in the pathogenesis of AMFB. Indeed, these tumors almost exclusively occur in women of reproductive age; two cases of postmenopausal women receiving tamoxifen therapy have been reported $[14,15]$. Because many of the histological and immunohistochemical features of AMFB, AA, and CA overlap, it has been suggested that these richly vascularized myxoid tumors of the genital tract represent members of the same fibroblasticmyofibroblastic tumor spectrum [16]. Sonography is a widely available, relatively cheap, extremely valuable tool to characterize and distinguish these tumors preoperatively and to plan surgery accordingly. In our opinion, MR imaging is not mandatory in the evaluation of soft tissue lesions of the lower genital tract such as AMFB in women. Postoperative pathological examination of the surgical specimen remains the only way to definitively establish a diagnosis of AMFB.

\section{Conclusions}

AMFB is a rare, benign stromal tumor arising most commonly from the female lower reproductive tract, and ultrasonography is the most important imaging modality in its preoperative diagnosis and management. AMFB appears as a homogeneous, well-defined, vascularized lesion of medium echogenicity. AMFB needs to be distinguished from aggressive angiomyxoma because the latter demands more extensive treatment.

\section{Consent}

Written informed consent was obtained from the patient for publication of this case report and any accompanying images. A copy of the written consent is available for review by the Editor-in-Chief of this journal. 


\section{Additional files}

Additional file 1: Ultrasonographic appearance of AMFB. Collection of short ultrasound clips showing the characteristics of AMFB. (WMV $11397 \mathrm{~kb}$ )

Additional file 2: Published case reports of AMFB up to the time of the writing of this report. (XLSX $21 \mathrm{~kb})$

\section{Abbreviations}

AA: aggressive angiomyoma; AF: angiofibroma; AMFB: Angiomyofibroblastoma; CA: cellular angiofibroma; H\&E: hematoxylin and eosin; MFB: myofibroblastoma; MR: magnetic resonance.

\section{Competing interests}

The authors declare that they have no competing interests.

\section{Authors' contributions}

BW wrote most of the manuscript, researched the literature, and did the statistical analysis of the previously published cases. LCH performed the histological examination of the tumor and provided the histological images. $\mathrm{RH}$ performed the ultrasound examinations. JE was the principal surgeon who cared for the patient and was much involved in writing the manuscript. All authors read and approved the final manuscript.

\section{Acknowledgments}

We thank the patient for her trust and cooperation in our publishing this case report.

\section{Author details}

${ }^{1}$ Department of Obstetrics and Gynecology, Leipzig University, Liebigstrasse 20a, 04103 Leipzig, Germany. ${ }^{2}$ Institute of Pathology, Leipzig University, Liebigstrasse 24, 04103 Leipzig, Germany.

Received: 5 January 2015 Accepted: 28 September 2015

Published online: 28 October 2015

\section{References}

1. Fletcher CD, Tsang WY, Fisher C, Lee KC, Chan JK. Angiomyofibroblastoma of the vulva: a benign neoplasm distinct from aggressive angiomyxoma. Am J Surg Pathol. 1992;16:373-82.

2. Poljak NK, Kljajić Z, Petricević J, Forempoher G, Simunić MM, Colović Z, et al. Polypoid angiomyofibroblastoma tumor of nasal cavity: case report. Coll Antropol. 2013:37:301-4

3. Sutton BJ, Laudadio J. Aggressive angiomyxoma. Arch Pathol Lab Med. 2012:136:217-21.

4. Fetsch JF, Laskin WB, Lefkowitz M, Kindblom LG, Meis-Kindblom JM. Aggressive angiomyxoma: a clinicopathologic study of 29 female patients. Cancer. 1996;78:79-90.

5. Chan YM, Hon E, Ngai SW, Ng TY, Wong LC, Chan IM. Aggressive angiomyxoma in females: is radical resection the only option? Acta Obstet Gynecol Scand. 2000:79:216-20.

6. Goodlad JR, Fletcher CD. Recent developments in soft tissue tumors. Histopathology. 1995;27:103-20

7. Magro G, Righi A, Casorzo L, Antonietta T, Salvatorelli L, Kacerovská D, et al. Mammary and vaginal myofibroblastomas are genetically related lesions: fluorescence in situ hybridization analysis shows deletion of 13 q14 region. Hum Pathol. 2012;43:1887-93.

8. Nielsen GP, Young RH, Dickersin GR, Rosenberg AE. Angiomyofibroblastoma of the vulva with sarcomatous transformation ("angiomyofibrosarcoma"). Am J Surg Pathol. 1997:21:1104-8.

9. Laiyemo R, Disu S, Vijaya G, Wise B. Post-menopausal vaginal angiomyofibroblastoma: a case report. Arch Gynecol Obstet. 2005;273:129-30.

10. Kavak ZN, Başgül A, Eren F, Ceyhan N. Angiomyofibroblastoma of the vulva: a rare but distinct entity. Acta Obstet Gynecol Scand. 2000:79:612-3.

11. Banerjee K, Datta Gupta S, Mathur SR. Vaginal angiomyofibroblastoma. Arch Gynecol Obstet. 2004:270:124-5.

12. Kim SW, Lee J, Han JK, Jeon S. Angiomyofibroblastoma of the vulva: sonographic and computed tomographic findings with pathologic correlation. J Ultrasound Med. 2009;28:1417-20.
13. Wang $X$, Yang $H$, Zhang $H$, Shi T, Ren W. Transvaginal sonographic features of perineal masses in the female lower urogenital tract: a retrospective study of 71 patients. Ultrasound Obstet Gynecol. 2014;43:702-10.

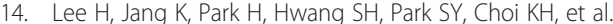
Angiomyofibroblastoma of the vagina in a breast cancer patient. Pathology. 2008:40:534-6.

15. Varras M, Akrivis C, Demou A, Kitsiou E, Antoniou N. Angiomyofibroblastoma of the vagina in a postmenopausal breast cancer patient treated with tamoxifen: clinicopathologic analysis of a case and review of the literature. Int J Gynecol Cancer. 2006;16:581-5.

16. Alameda F, Munné A, Baró T, Iglesias M, Condom E, Lloreta-Trull J, et al. Vulvar angiomyxoma, aggressive angiomyxoma, and angiomyofibroblastoma: an immunohistochemical and ultrastructural study. Ultrastruct Pathol. 2006:30:193-205

\section{Submit your next manuscript to BioMed Central and take full advantage of:}

- Convenient online submission

- Thorough peer review

- No space constraints or color figure charges

- Immediate publication on acceptance

- Inclusion in PubMed, CAS, Scopus and Google Scholar

- Research which is freely available for redistribution 